\title{
Material Contrast Information at the limit: Imaging of energy related materials with Backscattered Electrons obtained with Field Emission and the DELTA SEM
}

\author{
Ute Golla-Schindler ${ }^{1}$, Irene Wacker ${ }^{2}$, Bernd Schindler ${ }^{3}$, Ralf Löffler ${ }^{4}$, Dagmar Goll ${ }^{4}$, Gerhard Schneider ${ }^{4}$ and \\ Rasmus R. Schröder ${ }^{2}$
}

${ }^{1}$ 1. Materials Research Institute (IMFAA), Aalen University, Aalen, Germany, Aalen, Germany, ${ }^{2}$. Centre for Advanced Materials (CAM), University Heidelberg, Heidelberg, Germany, United States, ${ }^{3}$ Carl Zeiss Microscopy, Oberkochen, Germany, United States, ${ }^{4} 1$. Materials Research Institute (IMFAA), Aalen University, Aalen, Germany, United States

When detecting backscattered electrons, the attainable spatial resolution is improving with decreasing accelerating voltage due to the decrease of the interaction volume. One restriction is the loss of conventional material contrast information for detection energies below $1 \mathrm{keV}$ [1]. The conventional detection possibilities of backscattered electrons by semiconductor or scintillator detectors are limited to accelerating voltages above $3 \mathrm{kV}$. Further, the use of the ETD (Everhart-Thornley Detector) without suction power is restricted to small solid angles and, therefore, results in low signal to noise ratios. This restriction can be overcome with the inlens energy selective backscattered electron detector (EsB) or the DELTA Detector ${ }^{\circledR}$. The latter is integrated into a novel low-voltage SEM prototype, developed in the framework of the DELTA-project, based in Heidelberg [2]. This instrument allows correction of spherical and chromatic aberrations. Its newly designed DELTA Detector ${ }^{\circledR}$ employs two retarding grids improving energy resolution and has an increased detection efficiency due to an enlarged detection solid angle in comparison to the EsB, which provides a significant increase in image information and resolution [3]. Both detectors enable energy-filtered imaging, where ideally solely electrons with energies above the grid voltage contribute to the image.

Lithium-ion batteries (LIB) are one of the most prevalent electric storage systems. The formation of surface layers like the SEI (solid electrolyte interface) on the anode side and the CEI (cathode electrolyte interface) on the cathode side play an important role for the functionality and lifetime of a battery. The inspection of such surface layers is difficult and scanning electron microscopy is one of the key technologies to visualize those layers. The sample studied here was a cathode foil of a commercially available graphite/NMC (Li $\left[\mathrm{Co}_{1 / 3} \mathrm{Ni}_{1 / 3} \mathrm{Mn}_{1 / 3}\right] \mathrm{O}_{2}$ ) pouch cell of a cycled battery [4].

Fig. 1 shows (a) schematic drawing of the emitted energy spectrum and images recorded at $1 \mathrm{keV}$ landing energy with the DELTA Detector ${ }^{\circledR}$ with changing grid voltages of (b) $+100 \mathrm{~V}$, (c) $-10 \mathrm{~V},(\mathrm{~d})-50 \mathrm{~V}$, (e) $-700 \mathrm{~V}$ and (f) $-900 \mathrm{~V}$. The image information changes with decreasing grid voltage from topographical information (b) to pure material contrast information in (d-f). In (b) the typical cathode composites can be discriminated: The agglomeration of NMC particles (1), the binder phase (2) and the conductive additives (3). By definition, secondary electrons (SEs) are those with energies below $50 \mathrm{eV}$, with the main peak located around $10 \mathrm{eV}$. Due to this they can only leave the bulk material at surface-near regions, thus, creating the "edge effect" - bright rims visible at the edges and increased signal for tilted surfaces of the particles marked in (b) with arrowheads. With increasing grid voltage, the SEs are repelled and solely BSE contribute to the image. Thus, the information depth decreases with increasing grid voltage reducing the information depth from classical bulk material contrast (d) to surface material contrast information (e) and material contrast from the nm range by solely detecting the EREs (Elastically Reflected Electrons) (f). This allows to visualize with increasing contrast the CEI on top of the NMC particles (4) and nm sized precipitates (5).

Another necessity for obtaining material contrast information is to be above the detection limit. Permanent magnets based on Fe-Nd-B are important components in high efficiency traction motors for e-mobility and generators for renewable energy. There, cost reduction is a driving force for further developments, for instance substituting $\mathrm{Nd}$ by $\mathrm{Ce}$. FeNdCeB, which are sintered magnets produced by a dual alloy process, were analysed. Fig. 2 (a) shows a BSE image and the core shell structure of both components marked as P1 and P2 and (b) 
the correlated Ce element distribution image obtained with energy-dispersive x-ray spectroscopy (EDS). In the BSE image P1 has a dark core and a bright rim, while P2 shows inverse contrast. The Ce distribution image delivers for $\mathrm{P} 1$ a Ce enriched core and a rim with a lower Ce content and for P2 the opposite. Table 1 gives elemental formula, chemical composition, density and average atomic number for core and rim of both particles. The difference of the rim to core $\Delta_{(\mathrm{R}-\mathrm{C})}$ for $\mathrm{P} 1$ and core to rim $\Delta_{(\mathrm{C}-\mathrm{R})}$ for P2 deliver negative values for the density P1 $\Delta \rho_{(\mathrm{R}-\mathrm{C})}=-0,085$ and P2 $\Delta \rho_{(\mathrm{C}-\mathrm{R})}=-0,013$, which does not agree with the findings in the BSE image. However, the values of $\Delta \mathrm{Z}_{(\mathrm{R}-\mathrm{C})}=0,028$ and $\Delta \mathrm{Z}_{(\mathrm{C}-\mathrm{R})}=0,045$ of the average atomic number correlate with the BSE image. The atomic number or density dependence of material contrast is still under discussion. These experimental results show clearly that material contrast in SEM images is atomic number dependent. Additionally, we visualize atomic number differences up to $\Delta Z_{(\mathrm{R}-\mathrm{C})}=0,028$ approximately by a factor 3 lower than the expectation from literature.[5]
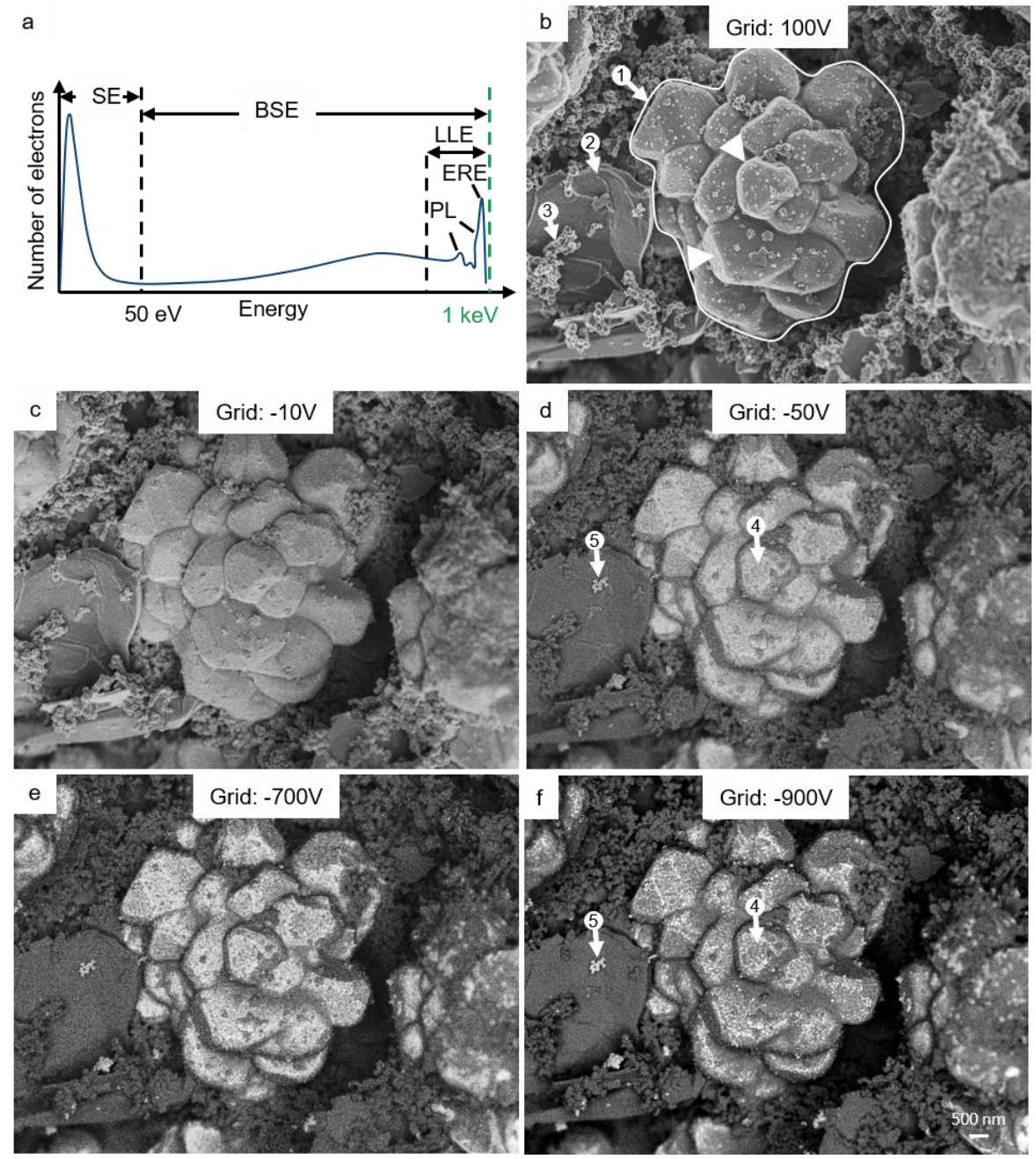

Figure 1. (a) Energy distribution of emitted electrons: Secondary Electrons (SE), Backscattered Electrons (BSE), Low Loss Electrons (LLE), Plasmon Losses (PL), Elastically Reflected Electrons (ERE). (b-f) Image series of agglomerated NMC particles (1) with binder phase (2) and conductive additives (3) of a cycled NMC cathode foil. Recorded with the DELTA detector ${ }^{\circledR}$ (DELTA-SEM) with grid voltages of (b) 100V, (c) -10 V, (d) $-50 \mathrm{~V}$ (e) $-700 \mathrm{~V}$ and (f) $-900 \mathrm{~V}$. 


\begin{tabular}{|c|c|c|c|c|c|c|}
\hline & & $\begin{array}{l}\text { Elemental } \\
\text { formular }\end{array}$ & $\begin{array}{l}\text { Density } \\
\text { g/lcm }^{3}\end{array}$ & & $\begin{array}{l}\text { Average } \\
\text { Atomic } \\
\text { number }\end{array}$ & \\
\hline \multirow[t]{2}{*}{ P 1} & Core & $\mathrm{Nd}_{1.24} \mathrm{Ce}_{0.76} \mathrm{Fe}_{14} \mathrm{~B}$ & 7,6137 & & 28,676 & \multirow{2}{*}{$\Delta Z_{(\mathrm{R}-\mathrm{C})}=0,028$} \\
\hline & Rim & $\mathrm{Nd}_{1.49} \mathrm{Ce}_{0.51} \mathrm{Fe}_{14} \mathrm{~B}$ & 7,6052 & & 28,704 & \\
\hline \multirow[t]{2}{*}{ P 2} & Core & $\mathrm{Nd}_{1.94} \mathrm{Ce}_{0.06} \mathrm{Fe}_{14} \mathrm{~B}$ & 7,5893 & & 28,758 & \multirow{2}{*}{$\Delta \mathrm{Z}_{(\mathrm{C}-\mathrm{R})}=0,045$} \\
\hline & Rim & $\mathrm{Nd}_{1.56} \mathrm{Ce}_{0.44} \mathrm{Fe}_{14} \mathrm{~B}$ & 7,602 & & 28,713 & \\
\hline
\end{tabular}

Table 1 Chemical composition of the core shell NdCeFeB particles P1 and P2

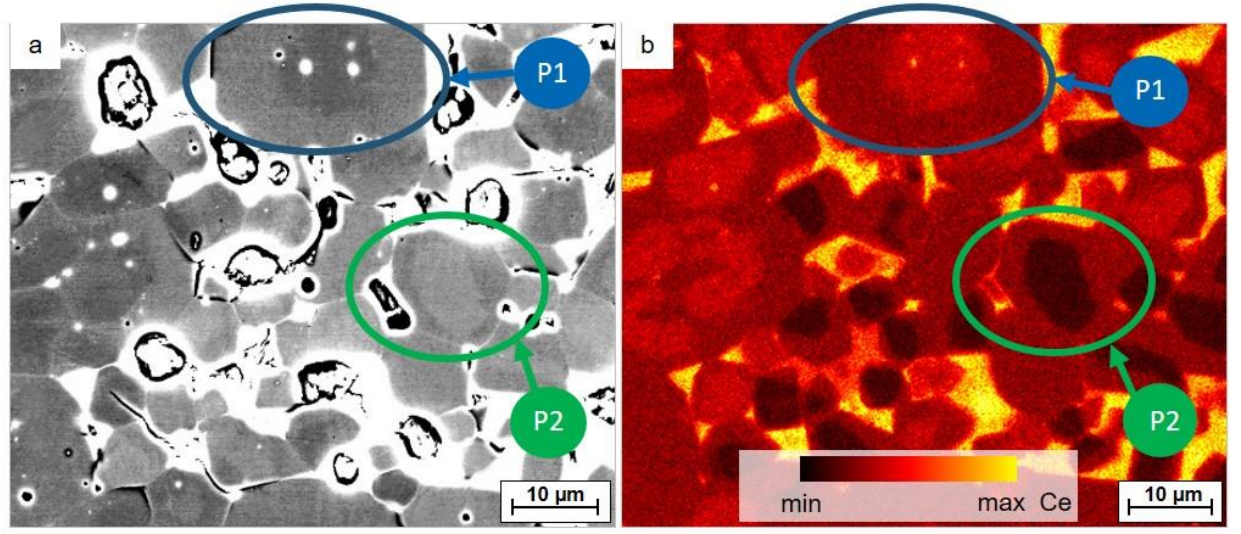

Figure 2. Images of $\mathrm{NdCeFeB}$ magnet particles with core shell structure: (a) BSE image with a 4 Quadrant semiconductor detector (b) Ce element distribution image with EDS mapping

\section{References}

[1] R. Böngeler et al., Scanning 15 (1993) p. 1-18

[2] R.R. Schröder et al., Microscopy and Microanalysis 24 (2018) p. 626

[3] U. Golla-Schindler et al., Microscopy and Microanalysis 25 (2019) p. 448

[4] U. Golla-Schindler et al., Micron 113 (2018) p. 10-19

[5] We thank the Centre for Advanced Materials and ZEISS GmbH for the DELTA images. I. Wacker is member and R,R.Schröder is PI of the Cluster of Excellence "3D Matter Made to Order" (EXC-2082/1 390761711) under Germany's Excellence Strategy. 